\title{
Unmasking Infective Endocarditis: A Case of Infective Spondylodiscitis
}

\author{
Nonyelum Obiechina $^{1 *}$, Nur Moud Foud ${ }^{1}$, Rachit Adlakha ${ }^{2}$, Serena Johal ${ }^{1}$ \\ ${ }^{1}$ University Hospitals of Derby and Burton NHS Foundation Trust, Queens Hospital, Belvedere Road, Burton \\ on-Trent, DE13 ORB, Staffordshire, UK \\ ${ }^{2}$ Health Education England, St Chads Court, Birmingham, West Midlands, B16 9RG, UK
}

*Corresponding Author: Dr Nonyelum Obiechina, Department of General Medicine and Geriatric Medicine, University Hospitals of Derby and Burton NHS trust, United Kingdom.

\begin{abstract}
Summary
Infective spondylodiscitis is a rare but serious infection of the vertebrae (osteomyelitis) with disc involvement (discitis).[1] It frequently presents with non-specific back pain, resulting in delayed diagnosis.[2] Most cases of spondylodiscitis occur via the haematogenous route from a distant septic focus including urinary tract infection, intravenous drug use and infective endocarditis.[3] Infective endocarditis is a rare, serious infection involving the endocardium and valves. Infected valves can cause septic embolization to the brain causing a brain abscess and to vertebral endplates causing osteomyelitis; this spreads contiguously to the discs causing spondylodiscitis.[3] As with infective spondylodiscitis it can occur sub-acutely with nonspecific symptoms.[4] We report the case of a 75-year-old woman who presented to the emergency department following several days of severe, non-traumatic back pain and acute urinary retention. She was found to have infective spondylodiscitis and blood cultures growing Streptococcus viridans and Streptococcus mitis, with transoesophageal echocardiogram confirming infective endocarditis.
\end{abstract}

Keywords: Infective Endocarditis, Valvar diseases, Musculoskeletal and joint disorders, infective spondylodiscitis

\section{BACKGROUND}

Infective spondylodiscitis is the infection of two adjacent vertebrae (spondylitis or osteomyelitis) and the disc between them (discitis).[1] The epidural or para-spinal region may also be affected resulting in epidural or para-spinal abscesses respectively.[2] This uncommon but serious infection is potentially life-threatening, with annual incidence in Europe estimated to be between 0.4 to 24 per 100,000 and is thought to be rising.[2] This may be due to people living longer, greater availability of diagnostic tools, and an increase in spinal surgical procedures.[2] Men are more affected than women with a ratio of between 1.5 - 2:1.[2] Some studies suggest a bimodal age distribution with peaks below the age of 20 and between 50 to 70 years of age.[2]

Most cases of spondylodiscitis arise from haematogenous spread from a distant focus of infection usually through bacteraemia from a urinary tract infection, wound infection, infected devices, or infective endocarditis.[3] Sometimes it may be caused by infection following spinal surgery such as a discectomy, with direct inoculation of the organisms at the time of surgery.[3] Predisposing factors to infective spondylodiscitis include cancer, diabetes mellitus, immunosuppression, older age, intravenous drug use.[3] Since infective endocarditis is a frequent source of infection in patients with spondylodiscitis, this diagnosis should prompt a search to confirm or exclude infective endocarditis.[4]

Infective endocarditis is also a rare and potentially fatal infection.[3][5-9] It is an inflammation of the endocardium, valves and interventricular septum caused mostly by blood borne infection from a bacteraemia, for instance, due to a dental abscess or a dental procedure.[3] Just like spondylodiscitis, infective endocarditis can present sub-acutely with very non-specific symptoms and signs.[3] As such, a high index of suspicion is needed to consider this as a differential diagnosis in the context of infective spondylodiscitis, particularly in patients with risk factors such as recent dental treatment, 
prosthetic heart valves and known history of valvular heart disease.[3] Untreated infective endocarditis has a high mortality and morbidity.[10] This case illustrates both the non-specific nature of presentation of both conditions, and how infective endocarditis can result in damage to the infected valves leading to a need for valve replacement.

\section{Case Presentation}

A 75-year-old woman presented to the emergency department following several days of severe, non- traumatic back pain and acute urinary retention. Codeine, diazepam, Entonox and paracetamol were administered in the emergency department and her background history of hypertension, well-controlled asthma, eczema and Raynaud's phenomenon were noted. On presentation, she was afebrile and her general examination was unremarkable. Systemic examination revealed a bilateral paraparesis, tenderness over the paraspinal area, and, evidence of urinary retention. She was suspected of having a spinal cord compression or cauda equina syndrome.

\subsection{Investigations}

Magnetic Resonance Imaging (MRI) of the spine (see figure 1) revealed high signal intensity at L2/L3 level and narrowed intervertebral disc space. Apart from an incidental finding of possible hemangioma to the vertebra, there was no evidence of cord compression, a corda equina lesion or discitis.

Her C-Reactive Protein (CRP) was raised. She was hyponatraemia and her bendroflumethiazide was discontinued. Her full blood count and other biochemical tests were normal. Her chest $\mathrm{x}$-ray and urine cultures came back normal. In view of her isolated raised CRP of unknown cause, a computed tomography (CT) scan of her abdomen and pelvis was arranged to see if she had an intra-abdominal or pelvic abscess. Although it did not show any intra-abdominal or pelvic collection, the CT scan showed evidence of osteomyelitis involving L2 and L3 vertebrae and discitis at the L2/L3 intervertebral disc (spondylodiscitis) (see figure 2).

At this point, she had blood cultures taken and was commenced on intravenous antibiotics. The first set of blood cultures grew streptococcus viridans and staphylococcus epidermidis, which the microbiologist commented may be due to a contaminant because of the polymicrobial nature of the findings. Subsequent blood cultures grew streptococcus mitis, a subspecies of streptococcus viridans, raising suspicion of infective endocarditis. Further questioning of the patient revealed that she had had her wisdom tooth removed three weeks prior to her admission.

A transthoracic echocardiogram (TTE) was arranged which showed mitral valve prolapse (posterior leaflet) with moderate eccentric regurgitation but did not reveal any vegetations; it was unable to completely rule out the presence of vegetations. It also showed a normal sized left ventricle and severely dilated left atrium. She was reviewed by the cardiologist who repeated the TTE a few weeks later; it showed marked mitral valve posterior leaflet calcification and an eccentric, anteriorly directed jet of severe regurgitation; again there were no vegetations present but it could not be completely ruled out. There was evidence of a mildly dilated left ventricle and severely dilated left atrium. She had a transoesophageal echocardiogram (TOE) which confirmed mitral valve regurgitation with likely vegetations (see figure 3). These findings were confirmed on histology after the patient underwent valve replacement.

\subsection{Figure/Video Captions}

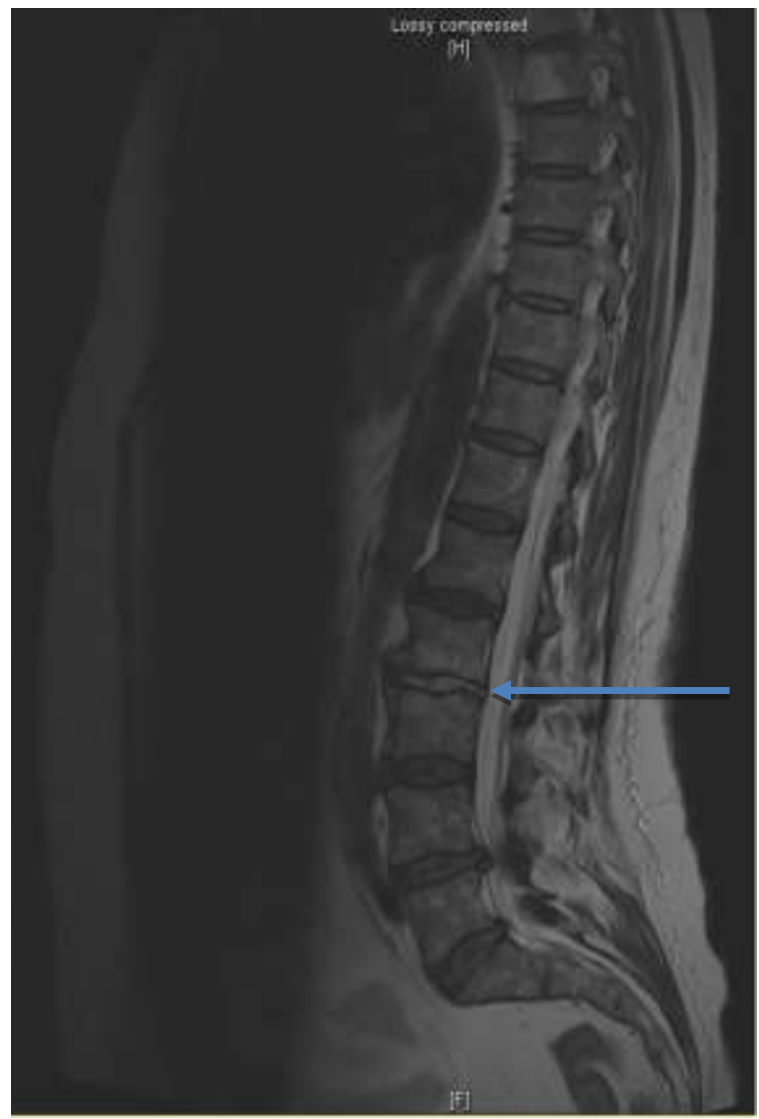

Figure1. MRI images - arrows pointing high intensity at L2/3 level and narrowed intervertebral disc space 


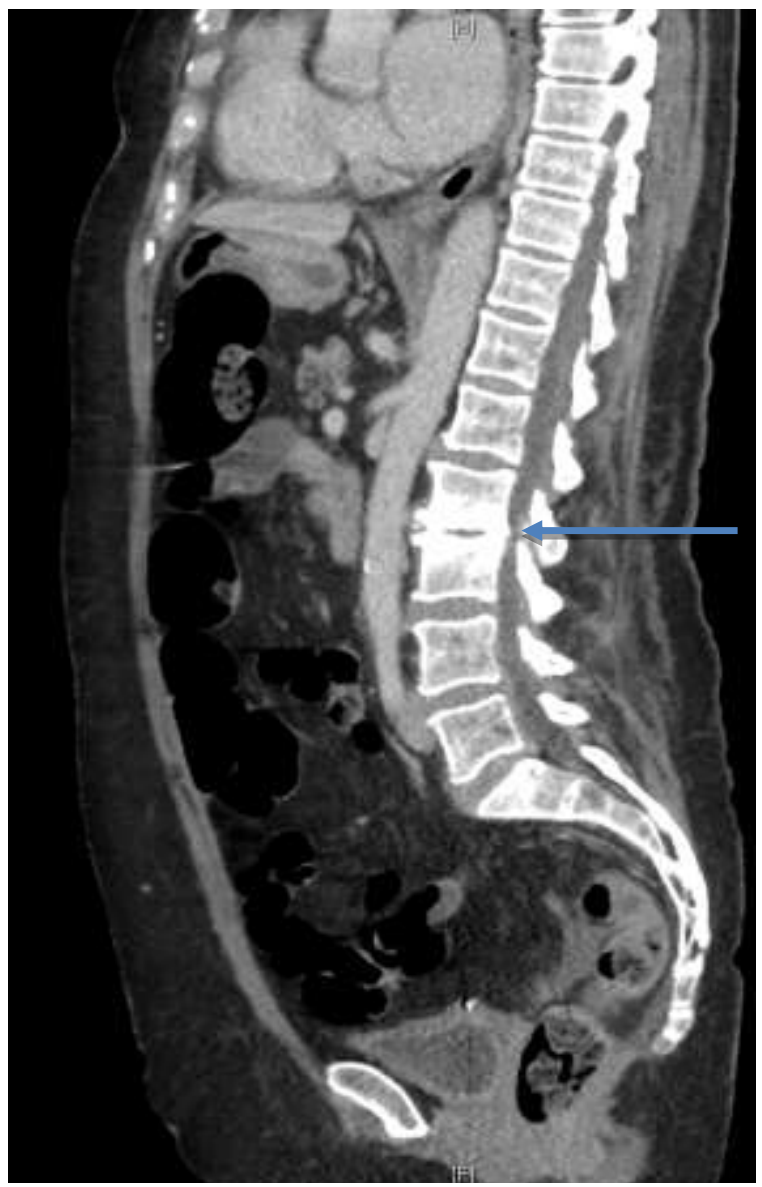

Figure2. CT Abdomen and Pelvis - arrows pointing at hyperintensity at L2/L3 level and narrowed intervertebral space

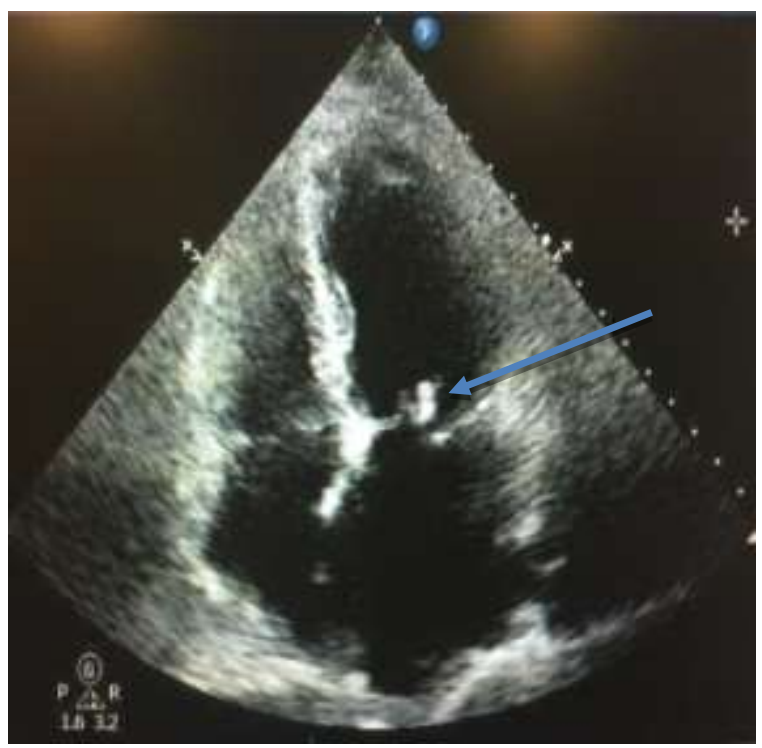

Figure3. Trans-oesophageal echocardiogramarrow pointing at vegetation over mitral valve

\subsection{Differential Diagnosis}

Differential diagnoses of spondylodiscitis include spinal cord compression, osteoporotic vertebral fractures and spinal malignancy. Spinal cord compression and cauda equina syndrome were initially suspected because of her paraparesis and urinary retention. Malignancy involving the spine can present with severe back pain and should be considered particularly if there are attendant red flags such as a previous history of malignancy, bladder or bowel dysfunction, saddle anaesthesia, reduced anal tone. Osteoporotic vertebral fracture can also cause severe back pain, although this tends to present as sudden onset back pain.

\subsection{Treatment}

Given the high clinical suspicion of infective endocarditis with positive blood cultures, the patient was treated with intravenous antibiotics for six weeks. During the course of her inpatient stay, she developed clinical features of decompensated heart failure secondary to severe mitral regurgitation. Following treatment of the heart failure, she was referred for mitral valve replacement. She was also reviewed by the maxillofacial surgeon prior to her mitral valve surgery and he was able to exclude a dental abscess.

\subsection{Outcome and Follow-Up}

The patient was transferred to a cardiac surgical unit where she underwent mitral valve replacement. She was discharged and is doing well with cardiac rehabilitation post-surgery. Histology of the lesions on the native valve confirmed vegetations.

\section{DISCUSSION}

The association between infective spondylodiscitis and infective endocarditis was first described and published by Seze and others in 1965.[11,12] Since then, other case series and case reports, as well as some observational studies have documented this association.[13 17]

\subsection{Epidemiology}

As previously stated, infective spondylodiscitis is an uncommon but serious infection which is potentially life-threatening.[2] Its annual incidence in Europe is estimated to be between 0.4 to 24 per 100,000 , but is thought to be rising.[2] This may be due to people living longer, greater availability of diagnostic tools and an increase in spinal surgical procedures.[3] Men are more affected than women with a ratio of between $1.5-2.1$.[3] Some studies suggest a bimodal age distribution with peaks below the age of 20 and between 50 to 70 years of age.[3]

Most cases of spondylodiscitis arise from haematogenous spread from a distant focus of 
infection, usually through bacteraemia from a urinary tract infection, wound infection, infected devices, or from infective endocarditis. $[3,4]$ Sometimes spondylodiscitis is caused by infection from direct inoculation during spinal surgery such as a discectomy.[3,18] Predisposing factors to infective spondylodiscitis include cancer, diabetes mellitus, immunosuppression, older age, and intravenous drug use. $[3,18]$

Infective endocarditis is also a rare, but serious condition. [4] Its incidence over the last 30 years has remained roughly two to six per 100,000 population.[4] In the United Kingdom (UK) annual incidence is thought to be 3 to 10 per 100,000 populations.[21] Men are more commonly affected than women.[22-25] In the pre-antibiotic era, rheumatic fever was the main predisposing factor for infective endocarditis and it was mainly common in children and young adults.[22 - 25] With the ageing of the population and better treatment and prevention of rheumatic heart disease, degenerative heart disease, prosthetic valves and cardiac devices such as pacemakers have become the more common predisposing factors for infective endocarditis.[25] Immunosuppression and intravenous drug use is, increasingly, contributing to the list of risk factors for infective endocarditis.[24,25]

The proportion of patients with infective spondylodiscitis who turn out to have underlying infective endocarditis and the percentage of infective endocarditis patients that develop spondylodiscitis have been studied and reported in various case reports and series and observational cohort studies. [12-17] Although these studies are limited by the small size of their cohorts, and lack of generalizability of their findings, they illustrate some interesting relationships between infective endocarditis and infective spondylodiscitis.

In a retrospective case-analysis Morelli and colleagues found that out of 30 cases of infective endocarditis that they reviewed, three had infective spondylodiscitis.[14] Conversely, Pigrau and others found that although only $4.6 \%$ of their 606 patients with infective endocarditis also had infective spondylodiscitis, $30.1 \%$ of their 91 patients with vertebral osteomyelitis turned out to have underlying infective endocarditis.[15] This comparatively high proportion of patients with spondylodiscitis, who turn out to have infective endocarditis, is echoed by other researchers.[16,17] The over- riding consensus among them is that patients who present with infective spondylodiscitis should be screened for infective endocarditis, both because of the high incidence of the latter, in this group of patients, and because of poorer outcomes when the two co-exist.[16,17]

\subsection{Pathogenesis and Pathophysiology of Infective Endocarditis and Spondylodiscitis}

Infective endocarditis frequently occurs in damaged valves and in the presence of bacteraemia.[15] Bacteraemia particularly, with oral commensals such as streptococcus viridans, may occur due to poor dental hygiene and /or in the context of invasive dental procedures.[18] Turbulence during blood flow through the valves causes exposure of the sub-endothelial structures to the circulating platelets.[20] These adhere to the sub-endothelium, aggregate and activate the coagulation cascade, via release of tissue thromboplastin, resulting in a platelet/fibrin thrombus plug.[20] This acts as a nidus for circulating bacteria, from bacteraemia, which adhere to the plug by secreting adhesins; further expansion of the clot leads to the bacteria walling themselves off and evading phagocytosis and opsonisation by the host immunological mechanisms.[20] This complex, known as a vegetation, may break off, as it expands, and embolize to the brain, spine, kidneys, and skin resulting in infective infarcts and abscesses as well as immune complex mediated damage such as occurs in the kidneys causing glomerulonephritis.[20]

These vegetations may embolize to the spinal arteries which, in adults, divide into two terminal arteries to the end plates of two adjacent vertebrae.[20] These terminal arteries are blocked by the vegetations, causing infarcts to the two adjacent vertebrae, and leading to secondary osteomyelitis.[20] The disc between the vertebrae is a vascular in adults but the infection spreads contiguously from the end plates of the adjoining vertebrae to the disc between them resulting in spondylodiscitis.[20] Common organisms implicated in these vegetations are frequently streptococcus viridans group and their subspecies such as streptococcus mitis and streptococcus mutans. [20] These are normally commensals in the mouth but attack damaged valves.[20] Staphylococcus aureus is one of the commonest causes of infective discitis.[26] It is, also, virulent enough to cause damage to normal valves, resulting in infective 
endocarditis.[26] This is more likely to occur in IVDU.[26] It may also infect prosthetic valves, as can staphylococcus epidermidis, which, though, frequently, a contaminant, can colonize, and cause infection in, prosthetic valves and

Table1. Clinical features of infective endocarditis related to causative organisms (adapted from: McDonald J.R. Acute Infective Endocarditis. Infect Dis Clin North Am 2009; 23(3):643 - 664). implanted cardiac devices such as pacemakers.[26] For a more comprehensive list of organisms implicated in infective endocarditis, see table 1.[27]

\begin{tabular}{|c|c|}
\hline Causative Organism(S) & Clinical Features of Infective Endocarditis \\
\hline Staphylococcus aureus & $\begin{array}{l}\text { - Overall, S aureus infection is the most common cause of IE, } \\
\text { including PVE, acute IE, and IVDA IE. } \\
\text { - Approximately 35-60.5\% of staphylococcal bacteremias are } \\
\text { complicated by IE. } \\
\text { More than half the cases are not associated with underlying } \\
\text { valvular disease. } \\
\text { - The mortality rate of } S \text { aureus IE is } 40-50 \% \text {. } \\
\text { - } \text { noureus infection is the second most common cause of } \\
\text { The incidence of MRSA infections, both the hospital- and } \\
\text { community-acquired varieties, has dramatically increased (50\% } \\
\text { of isolates). Sixty percent of individuals are intermittent carriers } \\
\text { of MRSA or MSSA. } \\
\text { The primary risk factor for } S \text { aureus BSI is the presence of } \\
\text { intravascular lines. Other risk factors include cancer, diabetes, } \\
\text { corticosteroid use, IVDA, alcoholism, and renal failure. } \\
\text { The realization that approximately 50\% of hospital- and } \\
\text { community-acquired staphylococcal bacteremias arise from } \\
\text { infected vascular catheters has led to the reclassification of } \\
\text { staphylococcal BSIs. BSIs are acquired not only in the hospital } \\
\text { but also in any type of health care facility (eg, nursing home, } \\
\text { dialysis center). } \\
\text { Of } S \text { aureus bacteremia cases in the United States, } 7.8 \% \text { (200,000) } \\
\text { per year are associated with intravascular catheters. }\end{array}$ \\
\hline Streptococcus viridans & $\begin{array}{l}\text { This organism accounts for approximately } 50-60 \% \text { of cases of } \\
\text { subacute disease. } \\
\text { - Most clinical signs and symptoms are mediated immunologically. }\end{array}$ \\
\hline \begin{tabular}{|l|}
$\begin{array}{l}\text { Streptococcus } \\
\text { intermedius group }\end{array}$ \\
\end{tabular} & $\begin{array}{l}\text { - These infections may be acute or subacute. } \\
\text { - } \text { intermedius infection accounts for } 15 \% \text { of streptococcal IE } \\
\text { cases. } \\
\text { Members of the } S \text { intermedius group, especially } S \text { anginosus, are } \\
\text { unique among the streptococci in that they can actively invade } \\
\text { tissue and form abscesses, often in the CNS. }\end{array}$ \\
\hline Abiotrophia & $\begin{array}{l}\text { Approximately } 5 \% \text { of subacute cases of IE are due to infection } \\
\text { with Abiotrophiaspecies. } \\
\text { - They require metabolically active forms of vitamin B-6 for } \\
\text { growth. } \\
\text { This type of IE is associated with large vegetations that lead to } \\
\text { embolization and a high rate of posttreatment relapse. }\end{array}$ \\
\hline Group D streptococci & $\begin{array}{l}\text { - Most cases are subacute. } \\
\text { - The source is the gastrointestinal or genitourinary tract. } \\
\text { - It is the third most common cause of IE. } \\
\text { - They pose major resistance problems for antibiotics. }\end{array}$ \\
\hline Nonenterococcal group D & $\begin{array}{l}\text { - The clinical course is subacute. } \\
\text { - Infection often reflects underlying abnormalities of the large } \\
\text { bowel (eg, ulcerative colitis, polyps, cancer). } \\
\text { - The organisms are sensitive to penicillin. }\end{array}$ \\
\hline
\end{tabular}




\begin{tabular}{|c|c|}
\hline Group B streptococci & $\begin{array}{l}\text { - Acute disease develops in pregnant patients and older patients } \\
\text { with underlying diseases (eg, cancer, diabetes, alcoholism). } \\
\text { - The mortality rate is } 40 \% \text {. } \\
\text { - Complications include metastatic infection, arterial thrombi, and } \\
\text { congestive heart failure. } \\
\text { - It often requires valve replacement for cure. }\end{array}$ \\
\hline $\begin{array}{l}\text { Group A, C, and G } \\
\text { streptococci }\end{array}$ & $\begin{array}{l}\text { - Acute disease resembles that of } S \text { aureus IE (30-70\% mortality } \\
\text { rate), with suppurative complications. } \\
\text { - Group A organisms respond to penicillin alone. } \\
\text { - Group C and G organisms require a combination of synergistic } \\
\text { antibiotics (as with enterococci). }\end{array}$ \\
\hline $\begin{array}{l}\text { Coagulase-negative } S \\
\text { aureus }\end{array}$ & $\begin{array}{l}\text { - This causes subacute disease. } \\
\text { - It behaves similarly to } S \text { viridansinfection. } \\
\text { - It accounts for approximately } 30 \% \text { of PVE cases and less than } 5 \% \\
\text { of NVE cases. }\end{array}$ \\
\hline $\begin{array}{l}\text { Staphylococcus } \\
\text { lugdunensis }\end{array}$ & $\begin{array}{l}\text { - Staphylococcus lugdunensis is another coagulase- } \\
\text { negative Staphylococcusspecies but is extremely aggressive } \\
\text { compared to coagulase-positive } S \text { aureus. } S \\
\text { lugdunensis frequently causes IE. }\end{array}$ \\
\hline Pseudomonas aeruginosa & $\begin{array}{l}\text { - This is usually acute, except when it involves the right side of the } \\
\text { heart in IVDA IE. } \\
\text { - Surgery is commonly required for cure. }\end{array}$ \\
\hline $\begin{array}{l}\text { HACEK (ie, Haemophilus } \\
\text { aphrophilus, Actinobacillus } \\
\text { actinomycetemcomitans, } \\
\text { Cardiobacterium hominis, } \\
\text { Eikenella corrodens, } \\
\text { Kingella kingae) }\end{array}$ & $\begin{array}{l}\text { - These organisms usually cause subacute disease. } \\
\text { - They account for approximately } 5 \% \text { of IE cases. } \\
\text { - They are the most common gram-negative organisms isolated } \\
\text { from patients with IE. } \\
\text { - Complications may include massive arterial emboli and } \\
\text { congestive heart failure. } \\
\text { - Cure requires ampicillin, gentamicin, and surgery. }\end{array}$ \\
\hline Fungal & $\begin{array}{l}\text { - These usually cause subacute disease. } \\
\text { - The most common organism of both fungal NVE and fungal PVE } \\
\text { is Candida albicans. } \\
\text { - Fungal IVDA IE is usually caused by Candida } \\
\text { parapsilosis or Candida tropicalis. } \\
\text { - Aspergillus species are observed in fungal PVE and NIE. }\end{array}$ \\
\hline Bartonella & $\begin{array}{l}\text { The most commonly involved species is Bartonella quintana. } \\
\text { IE typically develops in homeless males who have extremely } \\
\text { substandard hygiene. Bartonella must be considered in cases of } \\
\text { culture-negative endocarditis among homeless individuals. }\end{array}$ \\
\hline \begin{tabular}{|l} 
Multiple pathogens \\
(polymicrobial)
\end{tabular} & $\begin{array}{l}\text { - Pseudomonas and enterococci are the most common combination } \\
\text { of organisms. } \\
\text { - It is observed in cases of IVDA IE } \\
\text { - The cardiac surgery mortality rate is twice that associated with } \\
\text { single-agent IE. }\end{array}$ \\
\hline
\end{tabular}

\subsection{Clinical Presentation and Diagnosis}

The course of spondylodiscitis tends to be indolent and so its presentation can be very nonspecific, leading to delay in diagnosis and, in some cases, fatalities.[26] Severe back pain which is worse at night is frequently present and fever may occur but only in roughly half of patients.[26] Sometimes if an epidural or paraspinal abscess has occurred neurological signs of cord compression may result.[26] The diagnosis is usually made by radiological evidence of spondylodiscitis on a computerized tomography (CT) scan or a magnetic resonance imaging (MRI) scan and a positive blood culture.[26] Where the blood cultures are negative or equivocal, a CT-guided biopsy of the disc or vertebra may become necessary to make the diagnosis.[26]

Infective endocarditis is one of the sources of infections causing spondylodiscitis.[28] It may present acutely with pyrexia, a new murmur and/or congestive cardiac failure. In many cases, however, its presentation may be subacute and manifest with more non-specific 
findings such as lethargy, weight loss. Pyrexia may not always be present. The presence of a new cardiac murmur in this context should always alert one to the possibility of infective endocarditis. Three positive blood cultures as well as the presence of vegetations on cardiac valves on transthoracic (TTE) or transoesophageal echocardiography (TOE) usually makes the diagnosis likely. A history of recent dental infection or abscess and /or invasive dental treatment may frequently be present especially if the blood cultures. The presence of other predisposing factors such as known valvular disease or the presence of prosthetic heart valves also adds to the likelihood of the diagnosis. Definitive diagnosis is made histologically when the vegetations are removed during valve surgery and examined.[28]

It is worth bearing in mind that bacteraemia can cause infective endocarditis but infective endocarditis itself can also cause haematogenous infection to other organs such as the spine. This underscores the need to investigate the possibility of underlying infective endocarditis when bacteraemia and spondylodiscitis are identified.[28] It is also worth asking patients about their dental history as this may not always be volunteered automatically by the patients.

\subsection{Treatment}

Treatment of infective spondylodiscitis is usually with intravenous antibiotics for at least 6 weeks.[26] In some cases it may be necessary to drain a paraspinal abscess or emergency spinal surgery may be required for cord compression from an epidural abscess.[26] Treatment of infective endocarditis usually consists of at least 6 weeks of intensive intravenous antibiotic therapy.[28,29] In some cases, especially where there has been significant damage to the valves, antibiotic treatment would normally be followed by valve replacement.[29] Treatment of any dental abscesses or other infection is also important and a dentist or maxillofacial surgeon may need to be involved in the patient's management.[29]

Antibiotic prophylaxis, in patients at high risk of developing infective endocarditis, when undergoing invasive dental and other procedures remains controversial.[6][7][28] The National Institute of Health and Clinical Excellence (NICE) does not recommend routine antibiotic prophylaxis in patients at risk of developing infective endocarditis when they undergo invasive dental, gastroenterological and genitourinary procedures.[7] In their review of evidence on benefit versus risk with routine antibiotic prophylaxis, their general consensus was that there was not enough evidence to recommend this and there are risks such as anaphylaxis that could result from antibiotic treatment.[7] They do, however, suggest that patients at risk of infective endocarditis who develop an infective process should be screened for infective endocarditis.[7] The American Heart Association (AHA) recommend antibiotic prophylaxis only in patients at risk of endocarditis undergoing dental procedures which involve the manipulation of gingival tissue, respiratory tract procedures and those of them with infected skin.[6] The European Society of Cardiology (ESC) recommend antibiotic prophylaxis only when the high risk patient undergoes dental procedures involving the gingiva or periapical region of the teeth or in the presence of perforation of the oral mucosa.[28] Maintaining good oral hygiene is also important in reducing the risk of infective endocarditis. [28]

\subsection{Prognosis}

Mortality from infective spondylodiscitis ranges between 2 and $20 \%$ with higher mortality when diagnosis is delayed.[28] Infective endocarditis is associated with high mortality and morbidity despite advances in diagnosis and treatment.[28] In-hospital mortality from infective endocarditis is about $14 \%$ with higher levels of 20 to $30 \%$ in elderly patients. Mortality is highest in patients with infective spondylodiscitis who also have infective endocarditis.[29] However, early diagnosis with prompt treatment improves outcomes in these patients.[29]

\section{Learning Points/Take Home Messages}

1. Consider the possibility of spondylodiscitis in atraumatic back pain which is disproportionate to the clinical findings especially if it occurs with pyrexia and/or raised inflammatory markers.

2. Patients with infective spondylodiscitis should have an echocardiogram, especially if the blood cultures grow streptococcus viridans or staphylococcus aureus and/or the patient has risk factors for infective endocarditis such as valvular heart disease.

3. Immunocompromised patients and elderly patients may not always have attendant 
pyrexia but this should not stop the clinician from suspecting infective spondylodiscitis in a patient with atraumatic back pain and, unexplained, raised inflammatory markers.

4. Early diagnosis of infective spondylodiscitis and infective endocarditis and their prompt management is associated with better outcomes, making the case for a high index of suspicion in pertinent cases.

5. Untreated or delayed treatment of infective spondylodiscitis and infective endocarditis are associated with a high mortality and morbidity, especially, when the two conditions occur together.

6. Good oral hygiene is important in reducing the risk of infective endocarditis.

\section{REFERENCES}

[1] Sans N, Faruch M, Lapègue F, Ponsot A, Chiavassa H, Railhac J.J. Infection du rachis Spondylodiscites. Journal de Radiologie Diagnostique et Interventionnelle 2012;93(6) :551-560.

[2] Mavrogenis A.F, Megaloikonomos P.D, Igoumenou V.G, Panagopoulos G.N, Giannitsioti E, Papadopoulos A, Papagelopoulos P.J. Spondylodiscitis revisited. EFORT Open Rev 2017;2: 447-461.

[3] Skaf G.S, Domloj N.T, Fehlings M.G, Bouclaous C.H, Sabbagh A.S, Kanafani Z.A, Kanj S.S. Pyogenic spondylodiscitis: An overview. Journal of Infection and Public Health 2010; 3(1): 5-16

[4] McIntyre V, Butany J, Boles D, Shao T. Recurrent infective endocarditis causing heart valve failure: A case report. Human Pathology: Case Reports 2017; 10: 39-42

[5] Holland T.L, Baddour L.M, Bayer A.S, Hoen B, Miro, J.M, \& Fowler V.G Jr. Infective endocarditis. Nature Reviews Disease Primers 2016; 2: 16059.

[6] Baddour L.M et al. Infective Endocarditis in Adults: Diagnosis, Antimicrobial Therapy, and Management of Complications: A Scientific Statement for Healthcare Professionals from the American Heart Association endorsed by the Infectious Diseases Society of America. Circulation 2015; 132(15): 1435-1486.

[7] National Institute for Health and Care Excellence. Prophylaxis against infective endocarditis: antimicrobial prophylaxis against infective endocarditis in adults and children undergoing interventional procedures. NICE [Online] 2008 updated 2016 July. Clinical guideline CG64. Available from: https://www.nice.org.uk/guidance/cg64 [Accessed November 2019]

[8] Cahill T.J, Baddour L.M, Habib G, Hoen B, Salaun E, Pettersson G.B, Schafers H.J, Prendergast B.D. Challenges in infective endocarditis. J. Am. Coll. Cardiol 2017;69: 325-344

[9] Koslow M, Kuperstein R, Eshed I, Perelman M, Maor E, Sidi Y. The unique clinical features and outcome of infectious endocarditis and vertebral osteomyelitis co-infection. Am J Med 2014;127(7): e9-e15.

[10] Akiyama T., Chikuda H., Yasunaga H., Horiguchi H., Fushimi K., Saita K. Incidence and risk factors for mortality of vertebral osteomyelitis: A retrospective analysis using the Japanese diagnosis procedure combination database. BMJ Open 2013;3: e002412.

[11] Seze S. de, Ryckewaert A, Kahn M.F et al. L'endocarditis d' Osler em rhumatologie. Rev Rhum Mal Osteoartic 1965;32: 739 - 744

[12] Calderaro D, Gualandro DM, Yu PC, Marques AC, Puig LB, Caramelli B. Spondylodiscitis and infectious endocarditis: a round-trip to be considered. Braz J Infect Dis 2008 Dec; 12(6): 544-5.

[13] Luz A, Castro A, Ribeiro R, Bernardo L, Carvalho H, Bernardo A, Gomes L. Viridans streptococcus endocarditis associated with spondylodiscitis. Rev Port Cardiol 2004;23(5): 723-8.

[14] Morelli S, Carmenini E, Caporossi AP, Aguglia G, Bernardo ML, Gurgo AM. Spondylodiscitis and infective endocarditis: Case studies and review of the literature. Spine 2001; 26(5): 499500.

[15] Pigrau C, Almirante B, Flores X, Falco V, Rodriguez D, Gasser I, Villanueva C, Pahissa A. Spontaneous pyogenic vertebral osteomyelitis and endocarditis: incidence, risk factors, and outcome. The American Journal of Medicine 2005; 118(11): 1287e17-e24.

[16] Mulleman D, Philippe P, Senneville E, Costes C, Fages L, Deprez X, Flipo RM, Duquesnoiy B. Streptococcal and enterococcal spondylodiscitis. High incidence of infective endocarditis in 50 cases. J Rheumatology 2006; 33(1): 91-7.

[17] Behmanesh B, Gessler F, Schnoes K, Dubinski D, Won S, Konczalla J, Seifert V, Weise L, and Setzer M. Infective endocarditis in patients with pyogenic spondylodiscitis: implications for diagnosis and therapy. Neurosurg Focus 2019; 46(1): e2.

[18] Tufan, Muge \& kart koseoglu, Hamide \& Duygu, Ersozlu-Bozkirli \& Ozlem, Azap \& Tufan, Kadir \& Yucel, Eftal. (2010). Spondylodiscitis and endocarditis caused by $\mathrm{S}$. 
vestibularis. The Brazilian journal of infectious diseases: an official publication of the Brazilian Society of Infectious Diseases 2010;14(4): 3779.

[19] Choudhur M, Patel B.R, Patel M, Bashir T. Streptococcus viridans osteomyelitis and endocarditis following dental treatment: a case report. Cases Journal 2009;9(2): 6857.

[20] Wang $\mathrm{Y}$ and Wang A. Complications of Endocarditis: Recent Advances in Infective Endocarditis. IntechOpen 2013 [Online]. Available from: https://www.intechopen.com/books/recentadvances-in-infectiveendocarditis/complications-of-endocarditis [Accessed November 2019].

[21] Laura A Hughes, Andrew Epstein, Neeraj Prasad. Incidence and epidemiology of infective endocarditis from 2010 to 2017 in a rural UK hospital. Br J Cardiol 2019;26: 67-8.

[22] Slipczuk L, Codolosa JN, Davila CD et al. Infective endocarditis epidemiology over five decades: a systematic review. PLoS One 2014; 9(10): e111564.

[23] Dayer MJ, Jones S, Prendergast B, Baddour LM, Lockhart PB, Thornhill MH. An increase in the incidence of infective endocarditis in England since 2008: a secular trend interrupted time series analysis. The Lancet 2015; 385(9974): 1219-28.

[24] Sapico FL, Liquete JA, Sarma RJ. Bone and joint infections in patients with infective endocarditis: review of a 4-year experience. Clin Infect Dis 1996; 22:783-87.
[25] Yellapu V, Ackerman D, Longo, Stawicki S.P. Septic Embolism in Endocarditis: Anatomic and Pathophysiologic Considerations. Advanced Concepts in Endocarditis 2018 [Online]. Available from: https://www.intechopen.com/books/advancedconcepts-in-endocarditis/septic-embolism-inendocarditis-anatomic-and-pathophysiologicconsiderations [Accessed November 2019].

[26] Bernard L, Dinh A, Ghout I, et al. Antibiotic treatment for 6 weeks versus 12 weeks in patients with pyogenic vertebral osteomyelitis: an open-label, non-inferiority, randomised, controlled trial. The Lancet 2015;385(9971): 875-882.

[27] McDonald J.R. Acute Infective Endocarditis. Infect Dis Clin North Am 2009; 23(3): 643 664.

[28] Habib G Lancellotti P Antunes MJ Bongiorni MG Casalta JP Del Zotti F. 2015 ESC Guidelines for the management of infective endocarditis: The Task Force for the Management of Infective Endocarditis of the European Society of Cardiology (ESC). Endorsed by: European Association for CardioThoracic Surgery (EACTS), the European Association of Nuclear Medicine (EANM). Eur Heart J. 2015; 36(44): 3075 - 3128.

[29] Le Moal G, Roblot F, Paccalin M, Sosner P, Burucoa C, Roblot P, Becq-Giraudon B. Clinical and laboratory characteristics of infective endocarditis when associated with spondylodiscitis. Eur J Clin Microbiol Infect Dis 2002 Sep;21(9): 671-5.

Citation: Nonyelum Obiechina, Nur Moud Foud, Rachit Adlakha, Serena Johal. Unmasking Infective Endocarditis: A Case of Infective Spondylodiscitis. ARC Journal of Clinical Case Reports. 2020; 6(3):15-23. DOI:https://doi.org/10.20431/2455-9806.0603004.

Copyright: (C) 2020 Authors. This is an open-access article distributed under the terms of the Creative Commons Attribution License, which permits unrestricted use, distribution, and reproduction in any medium, provided the original author and source are credited. 